\title{
APPROXIMATION OF PERIODIC FUNCTIONS IN WEIGHTED ORLICZ SPACES
}

\author{
Yunus E. YILDIRIR
}

Balikesir University, Turkey

\begin{abstract}
In the present work we prove some direct theorems of the approximation theory in the weighted Orlicz spaces with weights satisfying so called Muckenhoupt's condition and we obtain some estimates for the deviation of a function in the weighted Orlicz spaces from the linear operators constructed on the basis of its Fourier series.
\end{abstract}

\section{INTRODUCTION AND MAIN RESULTS}

A convex and continuous function $\Phi:[0, \infty) \rightarrow[0, \infty)$ for which $\Phi(0)=0$, $\Phi(x)>0$ for $x>0$, and

$$
\lim _{x \rightarrow 0} \frac{\Phi(x)}{x}=0, \quad \lim _{x \rightarrow 0} \frac{\Phi(x)}{x}=\infty
$$

is called a Young function.

The complementary Young function $\psi$ of $\Phi$ is defined by

$$
\psi(y):=\max \{x y-\Phi(x): x \geq 0\}
$$

for $y \geq 0$.

A Young function $\Phi$ said to satisfy $\Delta_{2}$ condition $\left(\Phi \in \Delta_{2}\right)$ if there is a constant $c>0$ such that

$$
\Phi(2 x) \leq c \Phi(x)
$$

for all $x \in \mathbb{R}$.

A nonnegative function $\varphi:[0, \infty) \rightarrow[0, \infty)$ is said to be quasiconvex if there exists a convex Young function $\Phi$ and a constant $c \geq 1$ such that, for

2010 Mathematics Subject Classification. 41A10, 42A10.

Key words and phrases. Direct theorem, weighted Orlicz space, Muckenhoupt weight, modulus of smoothness. 
all $x \geq 0$

holds.

$$
\Phi(x) \leq \varphi(x) \leq \Phi(c x)
$$

Let $\mathbf{T}:=[-\pi, \pi]$. A measurable function $\omega: \mathbf{T} \rightarrow[0, \infty]$ is called a weight function if the set $\omega^{-1}(\{0, \infty\})$ has Lebesgue measure zero.

A $2 \pi$-periodic weight function $\omega$ belongs to the Muckenhoupt class $A_{p}$, $p>1$, if

$$
\left(\frac{1}{|I|} \int_{I} \omega(x) d x\right)\left(\frac{1}{|I|} \int_{I} \omega^{-1 /(p-1)}(x) d x\right)^{p-1} \leq C
$$

with a finite constant $C$ independent of $I$, where $I$ is any subinterval of $\mathbf{T}$ and $|I|$ denotes the length of $I$.

Let $\varphi$ be a quasiconvex Young function. We denote by $\tilde{L}_{\varphi, \omega}(\mathbf{T})$ the class of Lebesgue measurable functions $f: \mathbf{T} \rightarrow \mathbb{C}$ satisfying the condition

$$
\int_{\mathbf{T}} \varphi(|f(x)|) \omega(x) d x<\infty .
$$

The linear span of the weighted Orlicz class $\tilde{L}_{\varphi, \omega}(\mathbf{T})$, denoted by $L_{\varphi, \omega}(\mathbf{T})$, becomes a normed space with the Orlicz norm

$$
\|f\|_{\varphi, \omega}:=\sup \left\{\int_{\mathbf{T}}|f(x) g(x)| \omega(x) d x: \int_{\mathbf{T}} \psi(|g(x)|) d x \leq 1\right\},
$$

where $\psi$ is the complementary Young function of $\varphi$. We define the Luxemburg norm as

$$
\|f\|_{(\varphi, \omega)}:=\inf \left\{k>0: \int_{\mathbf{T}} \varphi\left(k^{-1}|f(x)|\right) \omega(x) d x \leq 1\right\} .
$$

There exist $([6$, p. 23] $)$ constants $c, C>0$ such that

$$
c\|f\|_{(\varphi, \omega)} \leq\|f\|_{\varphi, \omega} \leq C\|f\|_{(\varphi, \omega)} .
$$

For a quasiconvex function $\varphi$ we define the indice $p(\varphi)$ of $\varphi$ as

$$
\frac{1}{p(\varphi)}:=\inf \left\{\beta: \beta>0, \varphi^{\beta} \text { is quasiconvex }\right\}
$$

$([6$, p. 218]).

If $\omega \in A_{p(\varphi)}$, then it can be easily seen that $L_{\varphi, \omega}(\mathbf{T}) \subset L_{1}(\mathbf{T})$ and $L_{\varphi, \omega}(\mathbf{T})$ becomes a Banach space with the Orlicz norm. The Banach space $L_{\varphi, \omega}(\mathbf{T})$ is called weighted Orlicz space.

Throughout this paper, the constant $c$ denotes a generic constant, i.e., a constant whose values can change even between different occurrences in a chain of inequalities. 
Detailed information about Orlicz spaces, defined with respect to the convex Young function $\varphi$, can be found in [14]. Orlicz spaces, considered in this work, are investigated in the books [6] and [20].

Let $\varphi \in \Delta_{2}$ and $\varphi^{\theta}$ is quasiconvex for some $\theta \in(0,1)$. For $f \in L_{\varphi, \omega}(\mathbf{T})$ with $\omega \in A_{p(\varphi)}$, we define the shift operator $\sigma_{h}$ by

$$
\left(\sigma_{h} f\right)(x):=\frac{1}{2 h} \int_{-h}^{h} f(x+t) d t, \quad 0<h<\pi, \quad x \in T
$$

and the $r$-modulus of smoothness $(r=1,2, \ldots)$ by

$$
\Omega_{\varphi, \omega}^{r}(f, \delta):=\sup _{0<h_{i} \leq \delta}\left\|\prod_{i=1}^{r}\left(I-\sigma_{h_{i}}\right) f\right\|_{\varphi, \omega}, \quad \delta>0,
$$

where $I$ is the identity operator. This modulus of smoothness is well defined, because $\sigma_{h}$ is a bounded linear operator on $L_{\varphi, \omega}(\mathbf{T})$ under the conditions that $\varphi \in \Delta_{2}, \varphi^{\alpha}$ is quasiconvex for some $\alpha, 0<\alpha<1$, and $\omega \in A_{p(\varphi)}([1])$.

We define the shift operator $\sigma_{h}$ and the modulus of smoothness $\Omega_{\varphi, \omega}^{r}$ in this way, because the space $L_{\varphi, \omega}(\mathbf{T})$ is not, in general, invariant under the usual shift $f(x) \rightarrow f(x+h)$.

We denote by $E_{n}(f)_{\varphi, \omega}$ the best approximation of $f \in L_{\varphi, \omega}(\mathbf{T})$ by trigonometric polynomials of degree not exceeding $n$, i.e.,

$$
E_{n}(f)_{\varphi, \omega}=\inf \left\{\left\|f-T_{n}\right\|_{\varphi, \omega}: T_{n} \in \Pi_{n}, n=1,2, \ldots\right\},
$$

where $\Pi_{n}$ denotes the class of trigonometric polynomials of degree at most $n$. Note that the existence of $T_{n}^{*} \in \Pi_{n}$ such that

$$
E_{n}(f)_{\varphi, \omega}=\left\|f-T_{n}^{*}\right\|_{\varphi, \omega}
$$

follows, for example, from [4, p. 59, Th. 1.1].

Let also

$$
f(x) \sim \sum_{k=-\infty}^{\infty} c_{k} e^{i k x}=\sum_{k=0}^{\infty}\left(a_{k} \cos k x+b_{k} \sin k x\right)
$$

be the Fourier series of $f \in L^{1}(\mathbf{T})$. In addition, we put

$$
S_{n}(x, f):=\sum_{k=-n}^{n} c_{k} e^{i k x}=\sum_{k=0}^{n}\left(a_{k} \cos k x+b_{k} \sin k x\right), \quad n=0,1,2, \ldots
$$

Our main results formulated in the following enable us to make conclusions concerning the rate of vanishing of the quantities $R_{n}(f, \lambda)_{\varphi, \omega}$ and $R_{r}(f, \lambda)_{\varphi, \omega}$ by using the modulus of smoothness $\Omega_{\varphi, \omega}^{r}(f, \delta)$ and the best approximation $E_{n}(f)_{\varphi, \omega}$. In the theory of approximation, statements of this sort are called direct theorems. In this paper we investigate some direct theorems of approximation theory in the weighted Orlicz spaces and get a generalization of the results appeared in [17]. 
In the literature many results on such approximation problems have been obtained in weighted and non-weighted Lebesgue spaces. The corresponding results in the non-weighted Lebesgue spaces can be found in the books [4] and [22]. The best approximation problems by trigonometric polynomials in weighted Lebesgue spaces with weights belonging to the Muckenhoupt class were investigated in [7] and [15]. Detailed information on weighted polynomial approximation can be found in the books [5] and [16].

Approximation by trigonometric polynomials and other related problems in the Orlicz and weighted Orlicz spaces were studied in $[2,8-12,17,18,21,23$, $24]$.

Since every convex function is quasiconvex, the Orlicz spaces considered by us in this paper are more general than the Orlicz spaces studied in the above mentioned works. Therefore, the results obtained in this paper are new also in the non-weighted cases.

The relation $\preceq$ is defined as " $A \preceq B \Leftrightarrow$ there exists a constant $C$ such that $A \leq C B^{\prime \prime}$.

Our main results are the following.

Theorem 1.1. Let $f \in L_{\varphi, \omega}(\mathbf{T}), \varphi \in \Delta_{2}, \omega \in A_{p(\varphi)}$ and $\varphi^{\alpha}$ be quasiconvex for some $\alpha \in(0,1)$. For the system of numbers $\lambda_{\nu}^{(n)}=1-\left(\frac{\nu}{n}\right)^{2 r}$ $(\nu \leq n)$

$$
\begin{aligned}
R_{n}(f, \lambda)_{\varphi, \omega} & :=\left\|f(x)-\left[\frac{a_{0}}{2}+\sum_{\nu=1}^{n} \lambda_{\nu}^{(n)}\left(a_{\nu} \cos \nu x+b_{\nu} \sin \nu x\right)\right]\right\|_{\varphi, \omega} \\
& \leq C \Omega_{\varphi, \omega}^{r}\left(f, \frac{1}{n}\right) .
\end{aligned}
$$

Theorem 1.2. Let $\varphi \in \Delta_{2}, \omega \in A_{p(\varphi)}$ and $\varphi^{\alpha}$ be quasiconvex for some $\alpha \in(0,1)$. For a sequence of functions $\lambda_{\nu}(r)=1-\left(\nu\left|r-r_{0}\right|\right)^{2 k},\left(\nu \leq \frac{1}{\left|r-r_{0}\right|}\right)$ specified on some set $E$ of the real axis and in addition for any fixed $r \in E$ and any function $f \in L_{\varphi, \omega}$ the series

$$
\frac{a_{0}}{2}+\sum_{\nu=1}^{\infty} \lambda_{\nu}(r)\left(a_{\nu} \cos \nu x+b_{\nu} \sin \nu x\right)
$$

convergences to a metric of the space $L_{\varphi, \omega}(\mathbf{T})$, then

$$
\begin{aligned}
R_{r}(f, \lambda)_{\varphi, \omega} & :=\left\|f(x)-\left[\frac{a_{0}}{2}+\sum_{\nu=1}^{\infty} \lambda_{\nu}(r)\left(a_{\nu} \cos \nu x+b_{\nu} \sin \nu x\right)\right]\right\|_{\varphi, \omega} \\
& \leq C \Omega_{\varphi, \omega}^{k}\left(f,\left|r-r_{0}\right|\right) .
\end{aligned}
$$

Theorem 1.3. Let $f \in L_{\varphi, \omega}(\mathbf{T}), \varphi \in \Delta_{2}, \omega \in A_{p(\varphi)}$ and $\varphi\left(t^{\frac{1}{p_{0}}}\right)$ be a Young function for some $p_{0}>1$ satisfying the $\Delta_{2}$ condition and $\varphi^{\alpha}$ be 
quasiconvex for some $\alpha \in(0,1)$ such that

$$
\varphi(u v) \leq c \varphi(u) \varphi(v)
$$

with a constant $c>0$. Then for an arbitrary triangular matrix of the numbers $\left\{\lambda_{\nu}^{(n)}\right\}\left(\lambda_{0}^{(n)}=1, \lambda_{\nu}^{(n)}=0, \nu>n, n=0,1,2, \ldots\right)$

$$
R_{n}(f, \lambda)_{\varphi, \omega} \preceq\left\{\left[\sum_{\nu=0}^{m} E_{2^{\nu}-1}^{2}(f)_{\varphi, \omega} \delta_{2^{\nu}-1}^{2}\right]^{1 / 2}+E_{n}(f)_{\varphi, \omega}\right\}
$$

if $\varphi(\sqrt{u})$ is convex and

$$
R_{n}(f, \lambda)_{\varphi, \omega} \leq \inf _{k>0} \frac{1}{k}\left\{1+\sum_{\nu=0}^{m} \varphi\left[c k E_{2^{\nu}-1}(f)_{\varphi, \omega} \delta_{2^{\nu}-1}\right]\right\}+C E_{n}(f)_{\varphi, \omega}
$$

if $\varphi(\sqrt{u})$ is concave, where

$$
\begin{aligned}
\delta_{2^{r}, n} & :=\sum_{l=2^{r}}^{2^{r+1}-1}\left|\lambda_{\nu+1}^{(n)}-\lambda_{\nu}^{(n)}\right|+\left|1-\lambda_{2^{r}}^{(n)}\right|, \\
2^{m} & \leq n<2^{m+1} .
\end{aligned}
$$

The non-weighted analogues of these theorems were proved in [17].

\section{Auxiliary Result}

LeMma 2.1. Let $\varphi\left(t^{\frac{1}{p_{0}}}\right)$ be a Young function for some $p_{0}>1$ satisfying the $\Delta_{2}$ condition. Then there exists two positive constants $c_{1}$ and $c_{2}$ such that $c_{1} \int_{\mathbf{T}} \varphi(f(x)) \omega(x) d x \leq \int_{\mathbf{T}} \varphi\left(\left(\sum_{k=0}^{\infty} \delta_{k}^{2}\right)^{1 / 2}\right) \omega(x) d x \leq c_{2} \int_{\mathbf{T}} \varphi(f(x)) \omega(x) d x$ for arbitrary $f \in L^{1}(\mathbf{T}) \cap L_{\varphi}(\mathbf{T})$, where

$$
\delta_{k}:=\sum_{\nu=2^{k-1}}^{2^{k}-1} c_{\nu} e^{i \nu x}, \quad \delta_{0}:=\frac{1}{2} a_{0} .
$$

This lemma is proved in [13, Th. 2].

LEMma 2.2. Let $\varphi\left(t^{\frac{1}{p_{0}}}\right)$ be a Young function for some $p_{0}>1$ satisfying the $\Delta_{2}$ condition. Let $f_{n}(x)(n=1,2, \ldots)$ be a sequence of $2 \pi$ periodic functions in $L_{\varphi, \omega}(\mathbf{T}), \omega \in A_{p(\varphi)}$ and let $S_{n, k_{n}}(x)$ be the $k$-th partial sum of Fourier series of the function $f_{n}(x), k=k_{n}$ is a function of $n$. Then there exists a positive constant $C$ such that

$$
\int_{\mathbf{T}} \varphi\left(\left(\sum_{n=0}^{\infty}\left|S_{n, k_{n}}(x)\right|^{2}\right)^{1 / 2}\right) \omega(x) d x \leq C \int_{\mathbf{T}} \varphi\left(\left(\sum_{n=0}^{\infty}\left|f_{n}(x)\right|^{2}\right)^{1 / 2}\right) \omega(x) d x
$$


with a constant $C$ is independent of $f_{n}(x)$.

This lemma is proved in [13, Th. 1] by taking

$$
f:=\sum_{n=0}^{\infty}\left|S_{n, k_{n}}(x)\right|^{2} \quad \text { and } \quad g:=\sum_{n=0}^{\infty}\left|f_{n}(x)\right|^{2} .
$$

LEMma 2.3. Let $\lambda_{0}, \lambda_{1}, \ldots$ be a sequence of numbers such that

$$
\left|\lambda_{l}\right| \leq M, \quad \sum_{\nu=2^{l}}^{2^{l+1}-1}\left|\lambda_{\nu}-\lambda_{\nu+1}\right| \leq M \quad(l=0,1,2, \ldots) .
$$

Then the series

$$
a_{0} \lambda_{0} / 2+\sum_{\nu=0}^{\infty} \lambda_{\nu}\left(a_{\nu} \cos \nu x+b_{\nu} \sin \nu x\right),
$$

where $a_{\nu}, b_{\nu}$ are the Fourier coefficients of a function $f(x) \in L_{\varphi, \omega}[0,2 \pi]$, is a Fourier series of some function $h(x) \in L_{\varphi, \omega}[0,2 \pi]$ and the following inequality is valid:

$$
\int_{\mathbf{T}} \varphi(|h(x)|) \omega(x) d x \leq C \int_{\mathbf{T}} \varphi(|f(x)|) \omega(x) d x .
$$

This lemma is proved in a similar way as [23, Lemma 2.4].

\section{Proofs of MAin Results}

Proof of Theorem 1. Let $2^{m} \leq n<2^{m+1}$. By virtue of the property of the norm, we get

$$
\begin{aligned}
& \left\|f(x)-\left[\sum_{\nu=0}^{n} \lambda_{\nu}^{(n)} A_{\nu}(x)\right]\right\| \|_{\varphi, \omega} \\
& \quad \leq\left\|\sum_{\nu=0}^{n}\left(1-\lambda_{\nu}^{(n)}\right) A_{\nu}(x)\right\|_{\varphi, \omega}+\left\|\sum_{\nu=n+1}^{\infty} A_{\nu}(x)\right\|_{\varphi, \omega} \\
& \quad=I_{1}+I_{2},
\end{aligned}
$$

where $A_{\nu}(x):=a_{\nu} \cos \nu x+b_{\nu} \sin \nu x$. From [1, Lemma 3]

$$
\begin{aligned}
\left\|S_{n}(f, x)\right\|_{\varphi, \omega} & \preceq\|f(x)\|_{\varphi, \omega} \\
\left\|f(x)-S_{n}(f, x)\right\|_{\varphi, \omega} & \preceq E_{n}(f)_{\varphi, \omega} .
\end{aligned}
$$

It follows from the latter inequality that

$$
I_{2}=\left\|\sum_{\nu=n+1}^{\infty} A_{\nu}(x)\right\|_{\varphi, \omega} \preceq E_{n}(f)_{\varphi, \omega}
$$


and, since $([1$, Th. 2$])$

$$
E_{n}(f)_{\varphi, \omega} \preceq \Omega_{\varphi, \omega}^{r}\left(f, \frac{1}{n}\right)
$$

then

Now, we estimate

$$
\left\|\sum_{\nu=n+1}^{\infty} A_{\nu}(x)\right\|_{\varphi, \omega} \preceq \Omega_{\varphi, \omega}^{r}\left(f, \frac{1}{n}\right) .
$$

$$
I_{1}=\left\|\sum_{\nu=1}^{n} \frac{1-\lambda_{\nu}^{(n)}}{\left(1-\frac{\sin \frac{\nu}{n}}{\frac{\nu}{n}}\right)^{r}} A_{\nu}(x)\left(1-\frac{\sin \frac{\nu}{n}}{\frac{\nu}{n}}\right)^{r}\right\|_{\varphi, \omega}
$$

We let

$$
\mu_{\nu, r}^{(n)}=\left\{\begin{array}{ll}
\frac{1-\lambda_{\nu}^{(n)}}{\left(1-\frac{\sin \frac{\nu}{n}}{\frac{\nu}{n}}\right)^{r}}, & \text { for } \nu \leq n \\
0, & \text { for } \nu>n
\end{array}\right\}
$$

For the sequence $\left(\mu_{\nu, r}^{(n)}\right)$ the conditions of lemma 3 are fulfilled $([3])$. Then, according to lemma 3

$$
\begin{aligned}
I_{1} & =\left\|\sum_{\nu=1}^{n} \mu_{\nu, r}^{(n)} A_{\nu}(x)\left(1-\frac{\sin \frac{\nu}{n}}{\frac{\nu}{n}}\right)^{r}\right\|_{\varphi, \omega} \preceq\left\|\sum_{\nu=1}^{n} A_{\nu}(x)\left(1-\frac{\sin \frac{\nu}{n}}{\frac{\nu}{n}}\right)^{r}\right\|_{\varphi, \omega} \\
& \preceq \Omega_{\varphi, \omega}^{r}\left(f, \frac{1}{n}\right)
\end{aligned}
$$

and theorem 1 is proved.

Proof of TheOrem 2. By virtue of the property of the norm we get

$$
\begin{aligned}
R_{r}(f, \lambda)_{\varphi, \omega} \leq & \left\|\sum_{\nu=1}^{\left[\frac{1}{\left|r-r_{0}\right|}\right]}\left(1-\lambda_{\nu}(r)\right) A_{\nu}(x)\right\|_{\varphi, \omega} \\
& +\left\|\sum_{\nu=\left[\frac{1}{\left|r-r_{0}\right|}\right]+1}^{\infty}\left(1-\lambda_{\nu}(r)\right) A_{\nu}(x)\right\|_{\varphi, \omega} \\
= & I_{1}^{\prime}+I_{2}^{\prime} .
\end{aligned}
$$

We estimate

$$
I_{1}^{\prime}=\left\|\sum_{\nu=1}^{\left[\frac{1}{r-r_{0} \mid}\right]} \frac{1-\lambda_{\nu}(r)}{\left(1-\frac{\sin \nu\left|r-r_{0}\right|}{\nu\left|r-r_{0}\right|}\right)^{k}} A_{\nu}(x)\left(1-\frac{\sin \nu\left|r-r_{0}\right|}{\nu\left|r-r_{0}\right|}\right)^{k}\right\|_{\varphi, \omega}, \quad k=1,2, \ldots
$$


Let us assume

$$
\mu_{\nu, r}=\left\{\begin{array}{cl}
\frac{1-\lambda_{\nu}(r)}{\left(1-\frac{\sin \nu\left|r-r_{0}\right|}{\nu\left|r-r_{0}\right|}\right)^{k}}, & \text { for } \nu \leq\left[\frac{1}{\left|r-r_{0}\right|}\right], \\
0, & \text { for } \nu>n
\end{array}\right\} .
$$

For the sequence $\left\{\mu_{\nu, r}^{(n)}\right\}$ the conditions of lemma 3 are fulfilled. Consequently, according to lemma 3 , for $2^{m} \leq\left[\frac{1}{\left|r-r_{0}\right|}\right]<2^{m+1}$

$$
\begin{aligned}
I_{1}^{\prime} & \leq\left\|\sum_{\nu=1}^{2^{m+1}} \mu_{\nu, r} A_{\nu}(x)\left(1-\frac{\sin \nu\left|r-r_{0}\right|}{\nu\left|r-r_{0}\right|}\right)^{k}\right\|_{\varphi, \omega} \\
& \preceq\left\|\sum_{\nu=1}^{\infty} A_{\nu}(x)\left(1-\frac{\sin \nu\left|r-r_{0}\right|}{\nu\left|r-r_{0}\right|}\right)^{k}\right\|_{\varphi, \omega} \preceq \Omega_{\varphi, \omega}^{k}\left(f,\left|r-r_{0}\right|\right) .
\end{aligned}
$$

Let us now estimate $I_{2}^{\prime}$. It is easily seen that the conditions of lemma 3 are fulfilled for the system of numbers $\left\{1-\lambda_{\nu}(r)\right\}$. Then, according to lemma 3

$$
I_{2}^{\prime}=\left\|\sum_{\nu=\left[\frac{1}{\left|r-r_{0}\right|}\right]+1}^{\infty}\left(1-\lambda_{\nu}(r)\right) A_{\nu}(x)\right\|_{\varphi, \omega} \preceq \sum_{\nu=\left[\frac{1}{\left|r-r_{0}\right|}\right]+1}^{\infty} A_{\nu}(x) \|_{\varphi, \omega} .
$$

Hence, as in Theorem 1, it follows

$$
I_{2}^{\prime} \preceq \Omega_{\varphi, \omega}^{k}\left(f,\left|r-r_{0}\right|\right) .
$$

The latter completes the proof of the theorem.

Proof of TheOREM 3. Let $2^{m} \leq n<2^{m+1}$. We obtain by means of our previous argument the following

$$
\begin{aligned}
R_{n}(f, \lambda)_{\varphi, \omega} & =\left\|f(x)-\sum_{\nu=0}^{n} \lambda_{\nu}^{(n)} A_{\nu}(x)\right\|_{\varphi, \omega} \\
& \leq\left\|\sum_{\nu=1}^{n}\left(1-\lambda_{\nu}^{(n)}\right) A_{\nu}(x)\right\|_{\varphi, \omega}+\left\|\sum_{\nu=n+1}^{\infty} A_{\nu}(x)\right\|_{\varphi, \omega} \\
& \preceq\left\|\sum_{\nu=1}^{n}\left(1-\lambda_{\nu}^{(n)}\right) A_{\nu}(x)\right\|_{\varphi, \omega}+E_{n}(f)_{\varphi, \omega} .
\end{aligned}
$$


PERIODIC FUNCTIONS IN WEIGHTED ORLICZ SPACES

409

Let $\varphi(\sqrt{u})$ be a convex function. Bearing in mind the equivalence of the norms (1.1) and (1.2), by virtue of lemma 1, we have

$$
\begin{aligned}
& \left\|\sum_{\nu=1}^{n}\left(1-\lambda_{\nu}^{(n)}\right) A_{\nu}(x)\right\|_{(\varphi, \omega)} \\
& =\inf \left(k>0: \int_{\mathbf{T}} \varphi\left(k^{-1 \mid}\left|\sum_{\nu=1}^{n}\left(1-\lambda_{\nu}^{(n)}\right) A_{\nu}(x)\right|\right) \omega(x) d x \leq 1\right) \\
& \left.\preceq \inf \left(k>0: c_{\varphi} \int_{\mathbf{T}} \varphi\left(\left.k^{-1}\left|\sum_{\mu=0}^{m}\right| \sum_{\nu=2^{\mu}}^{2^{\mu+1}-1}\left(1-\lambda_{\nu}^{(n)}\right) A_{\nu}(x)\right|^{2}\right)^{\frac{1}{2}}\right) \omega(x) d x \leq 1\right) .
\end{aligned}
$$

Moreover, due to (1.3), the constant $D_{\varphi}$ may be chosen such that

$$
c_{\varphi} \varphi(u) \leq \varphi\left(D_{\varphi} u\right) .
$$

Then

$$
\begin{aligned}
& \left\|\sum_{\nu=1}^{n}\left(1-\lambda_{\nu}^{(n)}\right) A_{\nu}(x)\right\|_{(\varphi, \omega)} \\
& \quad \preceq \inf \left(k>0: \int_{0}^{2 \pi} \varphi\left(D_{\varphi} k^{-1}\left(\sum_{\mu=0}^{m} \sigma_{n, \mu}^{2}(x)\right)^{1 / 2}\right) \omega(x) d x \leq 1\right),
\end{aligned}
$$

where

$$
\sigma_{n, \mu}(x)=\sum_{\nu=2^{\mu}}^{2^{\mu+1}-1}\left(1-\lambda_{\nu}^{(n)}\right) A_{\nu}(x) .
$$

Let $\psi(u)=\varphi(\sqrt{u})$. Then

$$
\begin{aligned}
& \left\|\sum_{\nu=1}^{n}\left(1-\lambda_{\nu}^{(n)}\right) A_{\nu}(x)\right\|_{(\varphi, \omega)} \\
& \leq \inf \left(k>0: \int_{\mathbf{T}} \psi\left(D_{\varphi}^{2} k^{-2} \sum_{\mu=0}^{m} \sigma_{n, \mu}^{2}(x)\right) \omega(x) d x \leq 1\right) \\
& =\left[\inf \left(k>0: \int_{\mathbf{T}} \psi\left(D_{\varphi}^{2} k^{-1} \sum_{\mu=0}^{m} \sigma_{n, \mu}^{2}(x)\right) \omega(x) d x \leq 1\right)\right]^{\frac{1}{2}} \\
& =D_{\varphi}\left\|\sum_{\mu=0}^{m} \sigma_{n, \mu}^{2}(x)\right\|_{(\psi, \omega)}^{\frac{1}{2}} \leq D_{\varphi}\left[\sum_{\mu=0}^{m}\left\|\sigma_{n, \mu}^{2}(x)\right\|_{(\psi, \omega)}\right]^{\frac{1}{2}}=D_{\varphi}\left[\sum_{\mu=0}^{m}\left\|\sigma_{n, \mu}(x)\right\|_{(\psi, \omega)}^{2}\right]^{\frac{1}{2}},
\end{aligned}
$$


where

$$
\begin{aligned}
\left\|\sigma_{n, \mu}^{2}(x)\right\|_{(\psi, \omega)} & =\inf \left(k>0: \int_{\mathbf{T}} \psi\left(k^{-1} \sigma_{n, \mu}^{2}(x)\right) \omega(x) d x \leq 1\right) \\
& =\inf \left(k>0: \int_{\mathbf{T}} \varphi\left(k^{-1 / 2} \sigma_{n, \mu}(x)\right) \omega(x) d x \leq 1\right) \\
& =\inf \left(t^{2}>0: \int_{\mathbf{T}} \varphi\left(t^{-1} \sigma_{n, \mu}(x)\right) \omega(x) d x \leq 1\right) \\
& =\left\|\sigma_{n, \mu}(x)\right\|_{(\varphi, \omega)}^{2} .
\end{aligned}
$$

If we apply the Abel transform to $\Delta_{r, \sigma}$

$$
\begin{aligned}
\sigma_{n, \mu}(x)= & \sum_{\nu=2^{\mu}}^{2^{\mu+1}-1}\left(1-\lambda_{\nu}^{(n)}\right) A_{\nu}(x) \\
= & \sum_{\nu=2^{\mu}}^{2^{\mu+1}-1}\left(S_{\nu}(f, x)-S_{2^{\mu+1}-1}(f, x)\right)\left(\lambda_{\nu+1}^{(n)}-\lambda_{\nu}^{(n)}\right) \\
& +\left(S_{2^{\mu+1}-1}(f, x)-S_{2^{\mu}-1}(f, x)\right)\left(1-\lambda_{2^{\mu}}^{(n)}\right) .
\end{aligned}
$$

Then, by virtue of the inequality (3.1) and the monotonicity of the sequence of best approximations, we have

$$
\begin{aligned}
\left\|\sigma_{n, \mu}(x)\right\|_{(\varphi, \omega)} \leq & \sum_{\nu=2^{\mu}}^{2^{\mu+1}-1}\left\|S_{\nu}(f, x)-S_{2^{\mu+1}-1}(f, x)\right\|_{(\varphi, \omega)}\left|\lambda_{\nu+1}^{(n)}-\lambda_{\nu}^{(n)}\right|+ \\
& +\left\|S_{2^{\mu+1}-1}(f, x)-S_{2^{\mu}-1}(f, x)\right\|_{(\varphi, \omega)}\left|1-\lambda_{2^{\mu}}^{(n)}\right| \\
\preceq & E_{2^{\mu}-1}(f)_{\varphi, \omega} \delta_{2^{\mu}, n} .
\end{aligned}
$$

Then

$$
\left\|\sum_{\nu=1}^{n}\left(1-\lambda_{\nu}^{(n)}\right) A_{\nu}(x)\right\|_{(\varphi, \omega)} \leq D_{\varphi}\left(\sum_{\mu=0}^{m} E_{2^{\mu}-1}^{2}(f)_{\varphi, \omega} \delta_{2^{\mu}, n}^{2}\right)^{1 / 2} .
$$


Let $\varphi(\sqrt{u})$ be a concave function. In the inequality (3.1), we use the well-known formula for calculation of the (norm [14, p. 92]):

$$
\begin{aligned}
& \left\|\sum_{\nu=1}^{n}\left(1-\lambda_{\nu}^{(n)}\right) A_{\nu}(x)\right\|_{\varphi, \omega} \\
& \quad=\inf _{k>0} k^{-1}\left(1+\int_{\mathbf{T}} \varphi\left(k \sum_{\nu=1}^{n}\left(1-\lambda_{\nu}^{(n)}\right) A_{\nu}(x)\right) \omega(x) d x\right) .
\end{aligned}
$$

Applying lemma 1 and (3.2), we obtain

$$
\left\|\sum_{\nu=1}^{n}\left(1-\lambda_{\nu}^{(n)}\right) A_{\nu}(x)\right\|_{\varphi, \omega}=\inf _{k>0} k^{-1}\left(1+\int_{\mathbf{T}} \varphi\left(D_{\varphi}^{2} k^{2} \sum_{\mu=0}^{m} \sigma_{n, \mu}^{2}(x)\right)^{1 / 2} \omega(x) d x\right) .
$$

Since $\varphi(\sqrt{u})$ is concave, we have

$$
\left\|\sum_{\nu=1}^{n}\left(1-\lambda_{\nu}^{(n)}\right) A_{\nu}(x)\right\|_{\varphi, \omega}=\inf _{k>0} k^{-1}\left(1+\sum_{\mu=0}^{m} \int_{\mathbf{T}} \varphi\left(D_{\varphi} k \sigma_{n, \mu}(x)\right) \omega(x) d x\right) .
$$

Using the proof of lemma 9.2 in $[14$, p. 74$]$, it is easily seen that

$$
\int_{\mathbf{T}} \varphi\left[\frac{u(x)}{\|u(x)\|_{\varphi, \omega}}\right] \omega(x) d x \leq 1 .
$$

Using this inequality, (1.3) and (3.2)

$$
\begin{aligned}
\int_{\mathbf{T}} \varphi & \left(D_{\varphi} k \sigma_{n, \mu}(x)\right) \omega(x) d x \\
\quad & =c^{\prime}(\varphi) \int_{\mathbf{T}} \varphi\left(\frac{\sigma_{n, \mu}(x)}{\left\|\sigma_{n, \mu}(x)\right\|_{\varphi, \omega}}\right) \varphi\left(D_{\varphi} k\left\|\sigma_{n, \mu}(x)\right\|_{\varphi, \omega}\right) \omega(x) d x \\
& \leq \varphi\left(D_{\varphi}^{\prime} k\left\|\sigma_{n, \mu}(x)\right\|_{\varphi, \omega}\right) .
\end{aligned}
$$

Consequently, we obtain

$$
\begin{aligned}
\left\|\sum_{\nu=1}^{n}\left(1-\lambda_{\nu}^{(n)}\right) A_{\nu}(x)\right\|_{\varphi, \omega} & \leq \inf _{k>0} k^{-1}\left(1+\sum_{\mu=0}^{m} \varphi\left(D_{\varphi}^{\prime} k\left\|\sigma_{n, \mu}(x)\right\|_{\varphi, \omega}\right)\right) \\
& \leq \inf _{k>0} k^{-1}\left(1+\sum_{\mu=0}^{m} \varphi\left(D_{\varphi}^{\prime} k E_{2^{\mu}-1}(f)_{\varphi, \omega} \delta_{2^{\mu}, n}\right)\right) .
\end{aligned}
$$

This completes the proof. 


\section{REFERENCES}

[1] R. Akgun and D. M. Israfilov, Approximation in weighted Orlicz spaces, Math. Slovaca 61 (2011), 601-618.

[2] R. Akgun and D. M. Israfilov, Approximation and moduli of fractional order in Smirnov-Orlicz classes, Glas. Mat. Ser. III 43(63) (2008), 121-136.

[3] R. Akgun, Sharp Jackson and converse theorems of trigonometric approximation in weighted Lebesgue spaces, Proc. A. Razmadze Math. Inst. 152 (2010), 1-18.

[4] R. A. De Vore and G. G. Lorentz, Constructive approximation, Springer-Verlag, Berlin, 1993.

[5] Z. Ditzian and V. Totik, Moduli of smoothness, Springer-Verlag, New York, 1987.

[6] I. Genebashvili, A. Gogatishvili, V. Kokilashvili and M. Krbec, Weight theory for integral transforms on spaces of homogeneous type, Longman, Harlow, 1998.

[7] E. A. Haciyeva, Investigation of the properties of functions with quasimonotone Fourier coefficients in generalized Nikolskii-Besov spaces, Author's summary of dissertation, Tbilisi, 1986 (Russian).

[8] D. M. Israfilov and A. Guven, Approximation by trigonometric polynomials in weighted Orlicz spaces, Studia Math. 174 (2006), 147-168.

[9] D. M. Israfilov and R. Akgun, Approximation in weighted Smirnov-Orlicz classes, J. Math. Kyoto Univ. 46 (2006), 755-770.

[10] D. M. Israfilov, B. Oktay, and R. Akgun, Approximation in Smirnov- Orlicz classes, Glas. Mat. Ser. III 40(60) (2005), 87-102.

[11] V. M. Kokilashvili, On analytic functions of Smirnov-Orlicz spaces, Studia Math. 31 (1968), 43-59.

[12] V. M. Kokilashvili, A direct theorem on mean approximation of analytic functions by polynomials, Dokl. Akad. Nauk SSSR 10 (1969), 411-414.

[13] V. Kokilashvili, A note on extrapolation and modular inequalities, Proc. of A. Razmadze Math. Inst. 150 (2009), 91-97

[14] M. A. Krasnosel'skiı̆ and Ya. B. Rutickiŭ, Convex functions and Orlicz spaces, Noordhoff Ltd., Groningen, 1961.

[15] N. X. Ky, On approximation by trigonometric polynomials in $L_{u}^{p}$-spaces, Studia Sci. Math. Hungar. 28 (1993), 183-188.

[16] H. N. Mhaskar, Introduction to the theory weighted polynomial approximation, World Sci., River Edge, 1996.

[17] V. G. Ponomarenko, Approximation of periodic functions in Orlicz space, Translated from Sibirskii Matematicheskii Zhurnal 7 (1966), 1337-1346.

[18] A. R-K. Ramazanov, On approximation by polynomials and rational functions in Orlicz spaces, Anal. Math. 10 (1984), 117-132.

[19] M. M. Rao and Z. D. Ren, Application of Orlicz spaces, Dekker, 2002.

[20] M. M. Rao and Z. D. Ren, Theory of Orlicz spaces, Marcel Dekker, New Tork, 1991.

[21] K. Runovski, On Jackson type inequality in Orlicz classes, Rev. Mat. Complut. 14 (2001), 395-404.

[22] A. F. Timan, Theory of approximation of functions of a real variable, Pergamon press and Macmillan, Oxford, 1963.

[23] Y. E. Yildirir and D. M. Israfilov, The properties of convolution type transforms in weighted Orlicz spaces, Glas. Mat. Ser. III 45(65) (2010), 461-474.

[24] G. Wu, On approximation by polynomials in Orlicz spaces, Approx. Theory Appl. 7 (1991), 97-110. 
Y. E. Yildirir

Department of Mathematics

Faculty of Education

Balikesir University

10100, Balikesir

Turkey

E-mail: yildirir@balikesir.edu.tr

Received: 26.12.2011.

Revised: 7.4.2012. 